\title{
Reinforcement in an in vitro analog of appetitive classical conditioning of feeding behavior in Aplysia: Blockade by a dopamine antagonist
}

\author{
Fredy D. Reyes, Riccardo Mozzachiodi, Douglas A. Baxter, and John H. Byrne ${ }^{1}$ \\ W.M. Keck Center for Neurobiology of Learning and Memory, Department of Neurobiology and Anatomy, The University of Texas \\ Medical School at Houston, Houston, Texas 77030, USA
}

\begin{abstract}
In a recently developed in vitro analog of appetitive classical conditioning of feeding in Aplysia, the unconditioned stimulus (US) was electrical stimulation of the esophageal nerve (En). This nerve is rich in dopamine (DA)-containing processes, which suggests that DA mediates reinforcement during appetitive conditioning. To test this possibility, methylergonovine was used to antagonize DA receptors. Methylergonovine $(1 \mathrm{nM})$ blocked the pairing-specific increase in fictive feeding that is usually induced by in vitro classical conditioning. The present results and previous observation that methylergonovine also blocks the effects of contingent reinforcement in an in vitro analog of appetitive operant conditioning suggest that DA mediates reinforcement for appetitive associative conditioning of feeding in Aplysia.
\end{abstract}

The feeding behavior of Aplysia californica provides an attractive model system for investigating the cellular and molecular mechanisms underlying associative learning (see Susswein and Schwartz 1983; Colwill et al. 1997; Elliot and Susswein 2002; Katzoff et al. 2002; Brembs 2003; Brembs et al. 2004; Cropper et al. 2004). For example, aspects of feeding behavior (i.e., biting) can be modified by appetitive classical conditioning (Colwill et al. 1997; Lechner et al. 2000a); the isolated nervous system readily generates fictive feeding patterns (see Church and Lloyd 1994; Nargeot et al. 1997); neural correlates of conditioning can be identified and studied in the isolated nervous system (Lechner et al. 2000b; Lorenzetti et al. 2004); and in vitro analogs, which are amenable to cellular and molecular analyses, recapitulate behavioral and neural changes that follow in vivo training (Mozzachiodi et al. 2003; Brembs et al. 2004; Lorenzetti et al. 2004). Several lines of evidence indicate that the esophageal nerve (En) mediates the effects of the unconditioned stimulus (US) during appetitive classical conditioning. First, ingestion of food was used as the US during behavioral conditioning (Lechner et al. 2000a), and the ingestion of food is correlated with increased activity in En (Brembs et al. 2002). Second, lesions of En block conditioning (Lechner et al. 2000a). Finally, electrical stimulation of En during the in vitro analog of classical conditioning mimics the effects of the US that are produced by behavioral training (Mozzachiodi et al. 2003; Brembs et al. 2004; Lorenzetti et al. 2004). Histochemical analyses indicate that processes within En contain dopamine (DA) (Kabotyanski et al. 1998), which suggests that DA may mediate the effects of the US during appetitive classical conditioning. Therefore, the present study investigated whether methylergonovine, which is an antagonist of DA receptors (Ascher 1972; Drummond et al. 1980; Wright and Walker 1984; Buckett et al. 1990; Teyke et al. 1993; Nargeot et al. 1999c), blocks the acquisition of the pairing-specific changes in fictive feeding that are produced by the in vitro analog of classical conditioning.

The experimental procedures of the present study were iden-

'Corresponding author.

E-mail John.H.Byrne@uth.tmc.edu; fax (713) 500-0623.

Article and publication are at http://www.learnmem.org/cgi/doi/10.1101/ Im.92905. tical to those described previously (Mozzachiodi et al. 2003). Briefly, the buccal and cerebral ganglia were isolated with selected nerves attached. The ganglia were pinned to the floor of a Petri dish, which was coated with silicone elastomer (Sylgard, Dow Corning) and contained artificial seawater (ASW) with high concentrations of divalent cations (high divalent ASW) composed of (in mM) $210 \mathrm{NaCl}, 10 \mathrm{KCl}, 145 \mathrm{MgCl}_{2}, 20 \mathrm{MgSO}_{4}, 33$ $\mathrm{CaCl}_{2}$, and 10 HEPES (pH 7.5) (Mozzachiodi et al. 2003). The high divalent ASW was used to reduce neural activity while the ganglia and nerves were being positioned in the recording chamber (Byrne et al. 1978). The connective tissue sheath that covers the ganglia was left intact. After pinning the ganglia, platinum electrodes were positioned against appropriate nerves (see below) and isolated from the bath with petroleum jelly (Vaseline, Sherwood Medical). Then, the high divalent ASW was exchanged for control saline (i.e., normal ASW) or saline containing $1 \mathrm{nM}$ methylergonovine (Sigma Chemical). The composition of the control saline (in $\mathrm{mM}$ ) was as follows: $450 \mathrm{NaCl}, 10 \mathrm{KCl}, 10$ $\mathrm{CaCl}_{2}, 30 \mathrm{MgCl}_{2}, 20 \mathrm{MgSO}_{4}$, and 10 HEPES (pH 7.5). The preparations were maintained at $15^{\circ} \mathrm{C}$ by means of a bipolar temperature controller (Model MWS2107FL, Cambion Thermoelectric Products).

Conventional extracellular recording and stimulation techniques were used (Nargeot et al. 1997; Mozzachiodi et al. 2003). Electrical stimulation ( $4 \mathrm{sec}, 10 \mathrm{~Hz}, 0.5$-msec pulses) of the anterior branch of the right En (see Nargeot et al. 1997) was used as analog of the US. The US had approximately the same frequency and duration of electrical activity in the En that has been observed during feeding (Brembs et al. 2002). The CS was electrical stimulation (8 sec, $5 \mathrm{~Hz}, 0.5$-msec pulses) of the most medial and ventral branch of the right anterior tentacle nerve (AT4, see Lechner et al. 2000b). The frequency of AT4 stimulation used was similar to that recorded in vivo during mechanical stimulation of the tentacles (Anderson 1967; Fredman and Jahan-Parwar 1980). With the use of in vivo recordings from buccal nerves (Morton and Chiel 1993), it is possible to identify patterns of fictive feeding that are the neural correlates of consummatory feeding behavior. As in previous studies (Nargeot et al. 1997, 1999a,b; Lechner et al. 2000b; Mozzachiodi et al. 2003), patterns of fictive feeding (i.e., buccal motor programs [BMPs]) were monitored as 
coordinated bursts of large-unit activity in buccal nerves Bn2.1, $\mathrm{Rn} 1$, and I2 of the right buccal ganglion (for details, see Nargeot et al. 1997). The nerve signals were amplified with a differential AC amplifier (Model 1700, A-M Systems). Activity in the I 2 nerve corresponds to the protraction (P) of the radula, whereas activity in $\mathrm{Bn} 2.1$ corresponds to the retraction (R) of the radula, and large-unit activity in Rn.1 corresponds to closure (C) of the radula. The CS-evoked BMPs were classified as ingestion-like or rejection-like BMPs according to the criteria described in Nargeot et al. (1997). Only patterns that consisted of activity in all three buccal nerves clustered in a complete protraction/retraction cycle were classified as BMPs. Patterns consisting of trains of activity in only one or two of the three nerves were classified as incomplete patterns and were not included in the study.

A total of 81 preparations were used in this study. Control saline was used in one half of the preparations, and saline containing $1 \mathrm{nM}$ methylergonovine was used in the other half. Each preparation was assigned to one of four groups: paired or unpaired training in control saline or paired or unpaired training in the presence of methylergonovine. The experimenter was not aware of which solution was present in the bath. Thirty minutes after exchanging the high divalent ASW for control saline or saline containing $1 \mathrm{nM}$ methylergonovine, the intensity of the US was adjusted so that the US elicited a BMP, and the intensity of the CS was adjusted so that a single CS elicited only sporadic, nonpatterned neural activity. The intensities of the US and the CS were determined independently in each experiment based on the response of the preparation. Once the intensities of the CS and the US were set, they remained fixed throughout the remainder of the experiment. Approximately $10 \mathrm{~min}$ after adjusting the intensity of the US and the CS, four CSs were delivered with an interstimulus interval (ISI) of 1 min (i.e., Pre-test), and the total number of CS-elicited BMPs occurring during a 1-min period following each CS was counted. The total observation time of the Pre-test was $4 \mathrm{~min}$. Training began $10 \mathrm{~min}$ after the conclusion of the Pre-test. Two groups received either paired or unpaired training while bathed with control saline, whereas the other two groups received either paired or unpaired training in the presence of saline containing $1 \mathrm{nM}$ methylergonovine. In the paired training, the CS preceded the US with an ISI of $4 \mathrm{sec}$. In the unpaired protocol, the ISI between CS and US was 2 min. Training consisted of 10 trials with an intertrial interval of $4 \mathrm{~min}$. The experimenter, who was unaware of the type of solution used in a given experiment, counted the patterns. A second observer independently scored the data. To determine the reliability of the scoring process, the results obtained by the two observers were compared with the Kendall's coefficient of concordance (Zar 1999). The coefficient of concordance $(\mathrm{W}=0.963)$ indicated significant agreement between the two observers. Preparations were included in the present study only if the US elicited a BMP in at least half of the 10 training trials. Twenty-nine preparations failed to meet this criterion and were discarded. Of the discarded preparations, $55.2 \%$ were treated with control saline and $44.8 \%$ were treated with methylergonovine. Thus, the discarded preparations were evenly distributed between the group treated with control saline and the group treated with saline containing methylergonovine. Sixty minutes after the last conditioning trial, four CSs were presented with an ISI of $1 \mathrm{~min}$ (i.e., Post-test), and the number of CS-evoked BMPs occurring during a 1-min period following each CS was counted. The total observation time of the Post-test was $4 \mathrm{~min}$. The experiments were conducted in blocks of two preparations (i.e., paired training and unpaired training).

The effects of the training protocol were measured as the difference in the number of CS-evoked BMPs (i.e., Post-test minus Pre-test). During the Pre-test the responsiveness of the prepa- rations to the four CSs was very low. The mean $( \pm$ SEM) numbers of BMPs during the Pre-test were as follows: $0 \pm 0$ (Pre-test of paired group in control saline), $0.30 \pm 0.48$ (Pre-test of unpaired group in control saline), $0.23 \pm 0.59$ (Pre-test of paired group in methylergonovine), and $0.15 \pm 0.55$ (Pre-test of unpaired group in methylergonovine). No significant differences were observed in the number of BMPs that were evoked by the four CSs during Pre-tests across all four groups (Kruskal-Wallis one-way analysis of variance, $\mathrm{H}=5.45, \mathrm{DF}=3, P=0.161$ ), which indicates the ganglia in the four groups were comparable before the training began.

Although the effects of methylergonovine on Aplysia neurons have not been extensively characterized, several studies have shown that methylergonovine acts primarily as an antagonist of DA receptors (Ascher 1972; Drummond et al. 1980; Gospe and Wilson 1981; Teyke et al. 1993; Nargeot et al. 1999c; DíazRíos and Miller 2005). Nargeot et al. (1999c) examined the doseinhibition relationship between the bath concentration of methylergonovine and the expression of BMPs. High concentrations of methylergonovine (e.g., $1 \mu \mathrm{M}$ ) block the ability of preparations to produce BMPs, whereas low concentrations, such as 1 $\mathrm{nM}$, had very little effect on the baseline ability of preparations to generate BMPs. However, $1 \mathrm{nM}$ methylergonovine blocks associative changes in neural activity normally produced by contingent reinforcement in the in vitro analog of operant conditioning. We also found that $1 \mathrm{nM}$ methylergonovine had no detectable effect on the baseline properties of preparations. In the present study, $1 \mathrm{nM}$ methylergonovine had no effect on the response of preparations to the electrical shock that was used as the US. The intensity of the US sufficient to produce a BMP did not significantly differ among the unpaired and paired groups treated with either control saline or saline containing methylergonovine (Kruskal-Wallis one-way analysis of variance, $\mathrm{H}=1.059$, $\mathrm{DF}=3, P=0.787$ ) (Fig. $1 \mathrm{~A})$. The mean ( \pm SEM) values of the intensity of the US were as follows: $8.9 \pm 0.2 \mathrm{~V}$ (paired trained in control saline), $8.9 \pm 0.2 \mathrm{~V}$ (unpaired trained in control saline), $8.7 \pm 0.1 \mathrm{~V}$ (paired trained in methylergonovine), and $9.2 \pm 0.4 \mathrm{~V}$ (unpaired trained in methylergonovine). Similarly, $1 \mathrm{nM}$ methylergonovine had no effect on the response of preparations to the electrical shock that was used as the CS. No significant differences were observed in the intensity of the CS among the unpaired and paired groups treated with either solution (Kruskal-Wallis one-way analysis of variance, $\mathrm{H}=1.745$, $\mathrm{DF}=3, P=0.672$ ) (Fig. 1B). The intensities of the CS were as follows: $7.2 \pm 0.9 \mathrm{~V}$ (paired trained in control saline), $6.6 \pm 0.8$ $\mathrm{V}$ (unpaired trained in control saline), $7.5 \pm 0.7 \mathrm{~V}$ (paired trained in methylergonovine), and $7.3 \pm 1.5 \mathrm{~V}$ (unpaired trained in methylergonovine). Finally, no significant differences were found in the number of BMPs elicited during training among the paired and unpaired preparations treated with either control saline or saline containing methylergonovine (Kruskal-Wallis oneway analysis of variance, $\mathrm{H}=3.579, \mathrm{DF}=3, P=0.311$ ) (Fig. 1C). Therefore, methylergonovine did not change the responsiveness of the ganglia to electrical stimulation of either En or AT4.

Although methylergonovine had no effect on the responsiveness of the ganglia to the CS or the US, it prevented the acquisition of the pairing-specific increase in the number of CSevoked BMPs that is typically observed following in vitro classical conditioning (Mozzachiodi et al. 2003). Figure 2 illustrates representative recordings of CS-evoked BMPs in preparations that received paired and unpaired training while bathed either in control saline or in saline containing methylergonovine. In this example, a difference of two CS-evoked BMPs occurred between the Pre-test and the Post-test of the preparation trained with the paired protocol while in control saline (Fig. 2A). In contrast, no difference was observed in the number of CS-evoked BMPs in the 
A

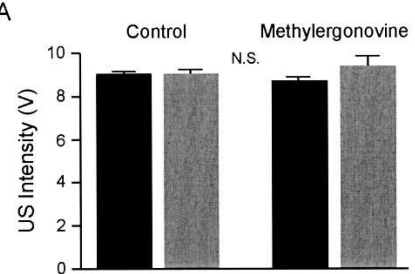

B
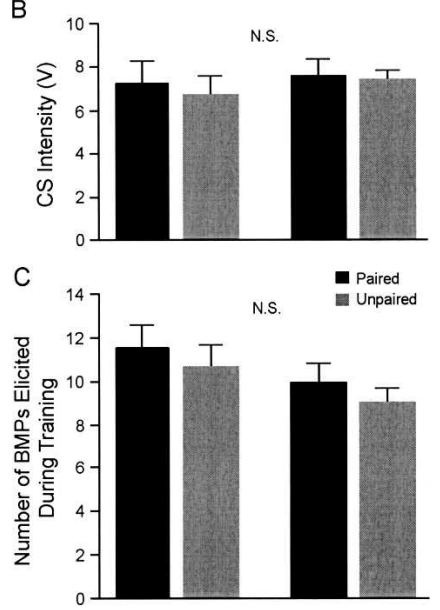

Figure 1. Effect of $1 \mathrm{nM}$ methylergonovine on the intensity of the electric shocks that were used for the US $(A)$ and CS $(B)$, and on the number of BMPs elicited during training $(C)$. (A) The intensity of stimulation that was used for the US (i.e., minimum En stimulus necessary to elicit a BMP) did not differ significantly among the four groups. (B) Similarly, methylergonovine had no effect on the minimum intensity of $\mathrm{AT}_{4}$ stimulation (i.e., the CS) that was necessary to elicit sporadic activity in the nerves. (C) No significant differences were found in the number of BMPs elicited during training among the paired and unpaired preparations treated with either control saline or saline containing methylergonovine. N.S. indicates that the difference was not statistically significant in this and subsequent figures.

preparation that received the paired training protocol while in the presence of methylergonovine (Fig. 2C). The number of CSevoked BMPs was not different between Pre-test and Post-test in preparations that received the unpaired training protocol independent of the solution (i.e., control saline or methylergonovine) (Fig. 2B,D). Thus, methylergonovine prevented the acquisition of conditioned changes in fictive feeding.

Summary data are presented in Figure 3. Statistical comparisons (i.e., Kruskal-Wallis one-way analysis of variance) indicated a significant difference in the effects of the training paradigms $(\mathrm{H}=21.469, \mathrm{DF}=3, P<0.001)$ that depended on the type of solution. Confirming Mozzachiodi et al. (2003), post hoc pairwise comparisons (i.e., Student-Newman-Keuls) indicated that in control saline the difference in the number of CS-evoked BMPs in the paired group was significantly greater than the difference in the number of CS-evoked BMPs in the unpaired group $(q=4.758$, $P<0.05)$. This pairing-specific enhancement demonstrated that classical conditioning occurred. In contrast, in the presence of methylergonovine, the difference in the number of CS-evoked BMPs in the paired group was not significantly different from that of preparations that received the unpaired training protocol $(q=2.321, P \geq 0.05)$. These data indicated that classical conditioning failed to occur in the presence of an antagonist of DA receptors. Moreover, the increase in the number of CS-evoked BMPs in the paired control group was significantly greater than the increase in the number of CS-evoked BMPs in the paired methylergonovine group $(q=5.584, P<0.05)$. This result indicated that methylergonovine impaired the associative increase in
CS-evoked fictive feeding produced by in vitro classical conditioning. Finally, the number of CS-evoked BMPs in the unpaired control group was not significantly different than the number of CS-evoked BMPs in the unpaired group treated with methylergonovine $(q=1.523, P \geq 0.05)$. Taken together, these results indicate that $1 \mathrm{nM}$ methylergonovine blocked the association between the CS and the US in the in vitro analog of classical conditioning.

In addition, the CS-evoked BMPs were classified. Because of the very low number of CS-evoked BMPs produced during the Pre-test (see above), we focused on the CS-evoked BMPs elicited during the Post-test. The number of CS-evoked ingestion-like BMPs during the Post-test was significantly different among the four groups of preparations (Kruskal-Wallis one-way analysis of variance, $\mathrm{H}=17.507, \mathrm{DF}=3, P<0.001)$. Post hoc pairwise comparisons (i.e., Student-Newman-Keuls) revealed that the number of CS-evoked ingestion-like BMPs in the paired group bathed with control saline was significantly greater when compared to the other three groups of preparations, which did not signifi-
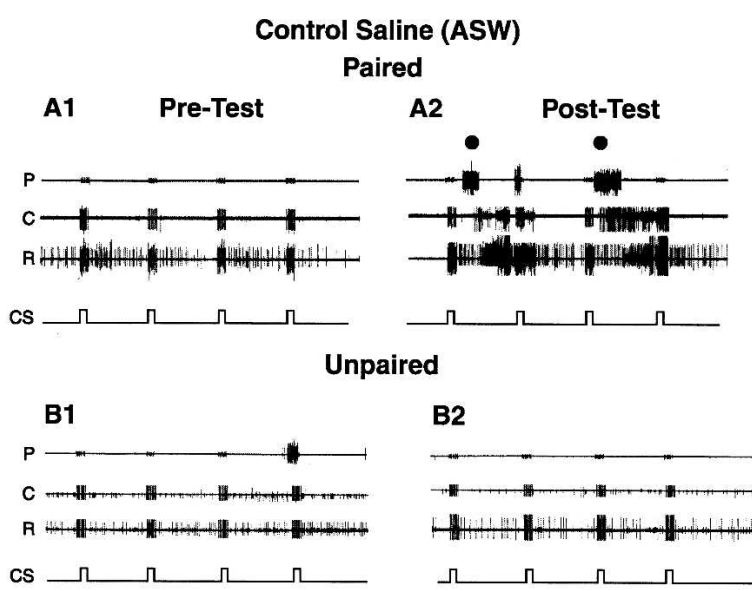

B2

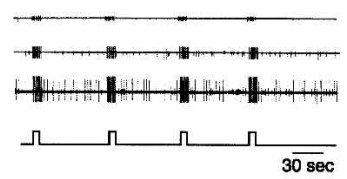

Methylergonovine (1 $\mathrm{nM}$ )

Paired
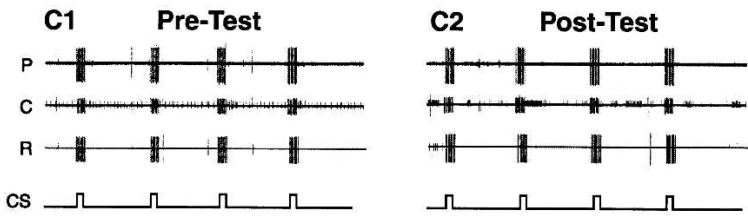

Unpaired

D1

D2
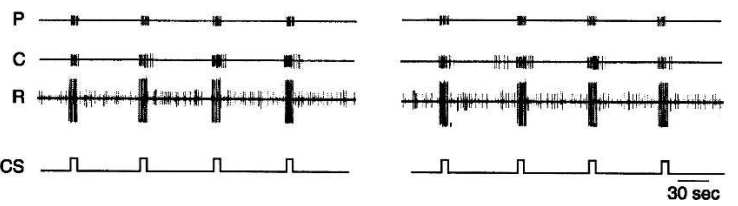

igure 2. Representative recordings before and after training in preparations that were bathed either in control saline $(A, B)$ or in saline containing $1 \mathrm{nM}$ methylergonovine $(C, D)$. The recordings illustrate the Pretest $(A 1, B 1, C 1, D 1)$ and Post-test $(A 2, B 2, C 2, D 2)$ presentations of the CS (lower traces). $\mathrm{P}$ indicates protraction; $\mathrm{C}$, closure; and $\mathrm{R}$, retraction. The filled circles indicate complete BMPs. Paired training in control saline produced an increase in the number of CS-evoked BMPs (A2), whereas unpaired training did not (B2). Paired training in the presence of methylergonovine did not lead to an increase in BMPs (C2), nor did unpaired training (D2). 


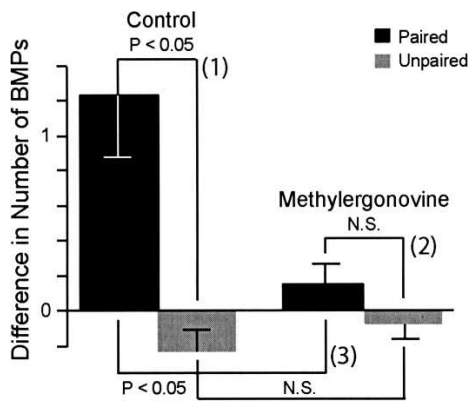

Figure 3. Methylergonovine prevented learning during the in vitro analog of classical conditioning. In control saline, paired training significantly increased the number of CS-evoked BMPs compared with preparations that received unpaired training (1). In methylergonovine, the number of CS-evoked BMPs after paired training was not significantly different from the number of CS-evoked BMPs after unpaired training (2). Finally, the number of CS-evoked BMPs from the paired group in the presence of methylergonovine was significantly less than those from the paired group in the presence of the control saline (3).

cantly differ from each other. These results confirm previous findings that the conditioning protocol was appetitive (Lechner et al. 2000b; Mozzachiodi et al. 2003).

DA is an important chemical transmitter in invertebrate nervous systems (see Gerschenfeld 1973; Gospe 1983; Goldstein and Schwartz 1989; Kabotyanski et al. 1998; Díaz-Ríos et al. 2002; Díaz-Ríos and Miller 2005). For example, several lines of evidence indicate that DA plays a role in appetitive operant conditioning (Nargeot et al. 1999c). Stimulation of En, which is rich in DAcontaining processes, can function as positive reinforcement during in vivo operant conditioning (Brembs et al. 2002). Similarly, in the in vitro analog of operant conditioning (Nargeot et al. 1997, 1999a,b), electrical stimulation of En delivered contingently with the expression of ingestion-like BMPs functions as a positive reinforcement. DA also mimics the effects of positive reinforcement in a single-cell analog of operant conditioning with cultured neuron B51 (Brembs et al. 2002). In all three preparations, contingent reinforcement produced an increase in the input resistance and a decrease in the threshold for plateau potential initiation in neuron B51 (Nargeot et al. 1999a,b). Finally, the contingent-dependent increase in the frequency of ingestionlike BMPs during in vitro operant conditioning was blocked when training occurred in the presence of $1 \mathrm{nM}$ methylergonovine (Nargeot et al. 1999c). Thus, DA appears to mediate reinforcement during appetitive operant conditioning.

The present study suggests that DA also mediates the effects of the US in appetitive classical conditioning. Methylergonovine, which acts as a DA-receptor antagonist, blocked the pairingspecific enhancement of the number of CS-evoked BMPs but did not change the responsiveness of the ganglia to the CS and US. We propose that the effects of methylergonovine on the change in the number of BMPs in the ganglia trained with the paired protocol were due to the blockade of the reinforcing effects mediated by dopaminergic transmission from the En. The possibility that methylergonovine may affect not only the acquisition but also the retrieval of conditioned changes in fictive feeding cannot be ruled out because the DA antagonist was bath-applied for the entire duration of the experiment. In addition, although the En is rich in DA-containing processes (Kabotyanski et al. 1998) and provides dopaminergic synaptic input to neurons such as B51 (Nargeot et al. 1999c), other dopaminergic neurons, including B20 and B65, are present in the feeding system (Teyke et al. 1993; Kabotyanski et al. 1998). These neurons also may be potential sites of action of the block of learning exerted by methylergonovine.
DA also plays a critical role in associative learning in mammals (Schultz 1997; Wise 2004; Liu et al. 2004). Pharmacological blockade of DA receptors interferes with the acquisition of conditioned behaviors produced by classical and operant conditioning procedures (Schultz 1997; Eyny and Horvitz 2003), and reinforcing stimuli activate dopaminergic neurons (Shizgal and Murray 1989; Schultz 2001). Moreover, the activity of dopaminergic neurons is correlated with changes in the parameters of reinforcing stimuli such as probability, uncertainty, and magnitude (Cromwell and Schultz 2003; Martin-Soelch et al. 2003; Fiorillo et al. 2003). The present results suggest that DA plays a similarly important role in associative learning in Aplysia. Feeding behavior of Aplysia provides a model system to future studies that will examine the cellular and molecular mechanisms of DA-mediated reinforcement.

\section{Acknowledgments}

We thank Dr. Gregg A. Phares for helpful comments on the manuscript. This work was supported by NIH grant MH058321.

\section{References}

Anderson, J.A. 1967. Patterns of response of neurons in the cerebral ganglion of Aplysia californica. Exp. Neurol. 19: 65-77.

Ascher, P. 1972. Inhibitory and excitatory effects of dopamine on Aplysia neurones. J. Physiol. (Lond.) 225: 173-209.

Brembs, B. 2003. Operant conditioning in invertebrates. Curr. Opin. Neurobiol. 13: $710-717$.

Brembs, B., Lorenzetti, F.D., Reyes, F.D., Baxter, D.A., and Byrne, J.H. 2002. Operant reward learning in Aplysia: Neuronal correlates and mechanisms. Science 296: 1706-1709.

Brembs, B., Baxter, D.A., and Byrne, J.H. 2004. Extending in vitro conditioning in Aplysia to analyze operant and classical processes in the same preparation. Learn. Mem. 11: 412-420.

Buckett, K.J., Dockray, G.J., Osborne, N.N., and Benjamin, P.R. 1990. Pharmacology of the myogenic heart of the pond snail Lymnaea stagnalis. J. Neurophysiol. 63: 1413-1425.

Byrne, J.H., Castellucci, V.F., and Kandel, E.R. 1978. Contribution of individual mechanoreceptor sensory neurons to defensive gill-withdrawal reflex in Aplysia. J. Neurophysiol. 41: 418-431.

Church, P.J. and Lloyd, P.E. 1994. Activity of multiple identified motor neurons recorded intracellularly during evoked feedinglike motor programs in Aplysia. J. Neurophysiol. 72: 1794-1809.

Colwill, R.M., Goodrum, K., and Martin, A. 1997. Pavlovian appetitive discriminative conditioning in Aplysia californica. Anim. Learn. Behav. 25: $268-276$.

Cromwell, H.C. and Schultz, W. 2003. Effects of expectations for different reward magnitudes on neuronal activity in primate striatum. J. Neurophysiol. 89: 2823-2838.

Cropper, E.C., Evans, C.G., Hurwitz, I., Jing, J., Proekt, A., Romero, A., and Rosen, S.C. 2004. Feeding neural networks in the mollusc Aplysia. Neurosignals 13: 70-86.

Díaz-Ríos, M. and Miller, M.W. 2005. Rapid dopaminergic signaling by interneurons that contain markers for catecholamines and GABA in the feeding circuit of Aplysia. J. Neurophysiol. 93: 2142-2156.

Díaz-Ríos, M., Oyola, E., and Miller, M.W. 2002. Colocalization of $\gamma$-aminobutyric acid-like immunoreactivity and catecholamines in the feeding network of Aplysia californica. J. Comp. Neurol. 445: $29-46$.

Drummond, A.H., Bucher, F., and Levitan, I.B. 1980. D- $\left[{ }^{3}\right.$ H]Lysergic acid diethylamide binding to serotonin receptors in the molluscan nervous system. J. Biol. Chem. 255: 6679-6686.

Elliott, C.J. and Susswein, A.J. 2002. Comparative neuroethology of feeding control in molluscs. J. Exp. Biol. 205: 877-896.

Eyny, Y.S. and Horvitz, J.C. 2003. Opposing roles of D1 and D2 receptors in appetitive conditioning. J. Neurosci. 23: 1584-1587.

Fiorillo, C.D., Tobler, P.N., and Schultz, W. 2003. Discrete coding of reward probability and uncertainty by dopamine neurons. Science 299: $1898-1902$.

Fredman, S.M. and Jahan-Parwar, B. 1980. Processing of chemosensory and mechanosensor information in identifiable Aplysia neurons. Comp. Biochem. Physiol. A 66: 25-34.

Gerschenfeld, H.M. 1973. Chemical transmission in invertebrate central nervous systems and neuromulscular junctions. Physiol. Rev. 53: $1-19$.

Goldstein, R.S. and Schwartz, J.H. 1989. Catecholamine neurons in Aplysia: Improved light-microscopic resolution and ultrastructural study using paraformaldehyde and glutaraldehyde (FaGlu) 
cytochemistry. J. Neurobiol. 20: 203-218.

Gospe, S.M. 1983. Studies of dopamine pharmacology in molluscs. Life Sci. 33: 1945-1957.

Gospe, S.M. and Wilson, W.A. 1981. Pharmacological studies of a novel dopamine-sensitive receptor mediating burst-firing inhibition of neurosecretory cell R15 in Aplysia californica. J. Pharmacol. Exp. Ther. 216: $368-377$.

Kabotyanski, E.A., Baxter, D.A., and Byrne, J.H. 1998. Identification and characterization of catecholaminergic neuron B65, which mediates and modifies patterned activity in the buccal ganglia of Aplysia. J. Neurophysiol. 79: 605-621.

Katzoff, A., Ben-Gedalya, T., and Susswein, A.J. 2002. Nitric oxide is necessary for multiple memory processes after learning that a food is inedible in Aplysia. J. Neurosci. 22: 9581-9594.

Lechner, H.A., Baxter, D.A., and Byrne, J.H. 2000a. Classical conditioning of feeding behavior in Aplysia, I: Behavioral analysis. J. Neurosci. 20: 3369-3376.

. 2000b. Classical conditioning of feeding behavior in Aplysia, II: Neurophysiological correlates. J. Neurosci. 20: 3377-3386.

Liu, Z., Richmond, B.J., Murray, E.A., Saunders, R.C., Steenrod, S., Stubblefield, B.K., Montague, D.M., and Ginns, E.I. 2004. DNA targeting of rhinal cortex D2 receptor protein reversibly blocks learning cues that predict reward. Proc. Natl. Acad. Sci. 101: $12336-12341$.

Lorenzetti, F.D., Mozzachiodi, R., Baxter, D.A., and Byrne, J.H. 2004 Both in vivo and in vitro classical conditioning of feeding enhance CS-evoked excitatory drive to B51 in Aplysia. Abstract

Viewer/Itinerary Planner, Program no. 778.7. 2004. Society for Neuroscience, Washington D.C.

Martin-Soelch, C., Missimer, J., Leenders, K.L., and Schultz, W. 2003. Neural activity related to the processing of increasing monetary reward in smokers and nonsmokers. Eur. J. Neurosci. 18: 680-688.

Morton, D.W. and Chiel, H.J. 1993. In vivo buccal nerve activity that distinguishes ingestion from rejection can be used to predict behavioral transitions in Aplysia. J. Comp. Physiol. A 172: 17-32.

Mozzachiodi, R., Lechner, H., Baxter, D.A., and Byrne, J.H. 2003. In vitro analog of classical conditioning of feeding behavior in Aplysia. Learn. Mem. 10: 478-494.
Nargeot, R., Baxter, D.A., and Byrne, J.H. 1997. Contingent-dependent enhancement of rhythmic motor pattern: An in vitro analog of operant conditioning. J. Neurosci. 17: 8093-8105.

. 1999a. In vitro analog of operant conditioning in Aplysia, I: Contingent reinforcement modifies the functional dynamics of an identified neuron. J. Neurosci. 19: 2247-2260.

. 1999b. In vitro analog of operant conditioning in Aplysia, II: Modifications of the functional dynamics of an identified neuron contribute to motor pattern selection. J. Neurosci. 19: 2261-2272.

Nargeot, R., Baxter, D.A., Patterson, G.W., and Byrne, J.H. 1999c. Dopaminergic synapses mediate neuronal changes in an analogue of operant conditioning. J. Neurophysiol. 81: 1983-1987.

Schultz, W. 1997. Dopamine neurons and their role in reward mechanisms. Curr. Opin. Neurobiol. 7: 191-197.

. 2001. Reward signaling by dopamine neurons. Neuroscientist 7: 293-302.

Shizgal, P. and Murray, B. 1989. Neuronal basis of intracranial self-stimulation. In The neuropharmacological basis of reward (eds. J.M. Liebman and S.J. Cooper), pp. 106-163. Oxford University Press, New York.

Susswein, A.J. and Schwartz, M. 1983. A learned change of response to inedible food in Aplysia. Behav. Neural. Biol. 39: 1-6.

Teyke, T., Rosen, S.C., Weiss, K.R., and Kupfermann, I. 1993. Dopaminergic neuron B20 generates rhythmic neuronal activity in the feeding motor circuitry of Aplysia. Brain Res. 630: 226-237.

Wise, R.A. 2004. Dopamine, learning and motivation. Nat. Rev. Neurosci. 5: $1-12$.

Wright, N.J.D. and Walker, R.J. 1984. The possible site of action of 5-hydroxytryptamine, 6-hydroxytryptamine, tryptamine and dopamine on identified neurons in the central nervous system of the snail Helix aspersa. Comp. Biochem. Physiol. C Toxicol. Pharmacol. 78: $217-225$.

Zar, J.H. 1999. Biostatistical analysis, 4th ed. Prentice-Hall, Engelwood Cliffs, NJ.

Received February 1, 2005; accepted in revised form March 29, 2005.

\section{Learning \& Memory \\ www.learnmem.org}




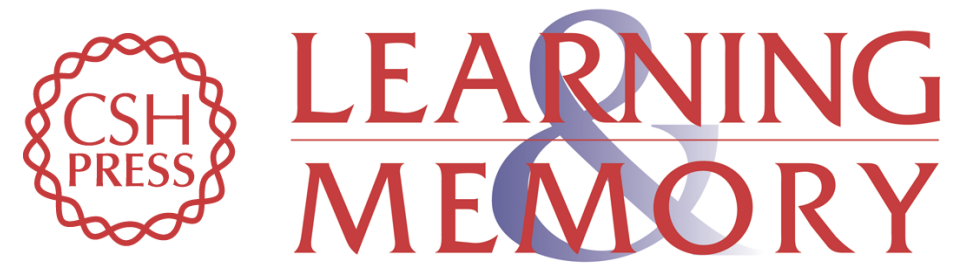

\section{Reinforcement in an in vitro analog of appetitive classical conditioning of feeding behavior in Aplysia: Blockade by a dopamine antagonist}

Fredy D. Reyes, Riccardo Mozzachiodi, Douglas A. Baxter, et al.

Learn. Mem. 2005, 12:

Access the most recent version at doi:10.1101//m.92905

References This article cites 39 articles, 15 of which can be accessed free at: http://learnmem.cshlp.org/content/12/3/216.full.html\#ref-list-1

License

Email Alerting Receive free email alerts when new articles cite this article - sign up in the box at the Service top right corner of the article or click here. 